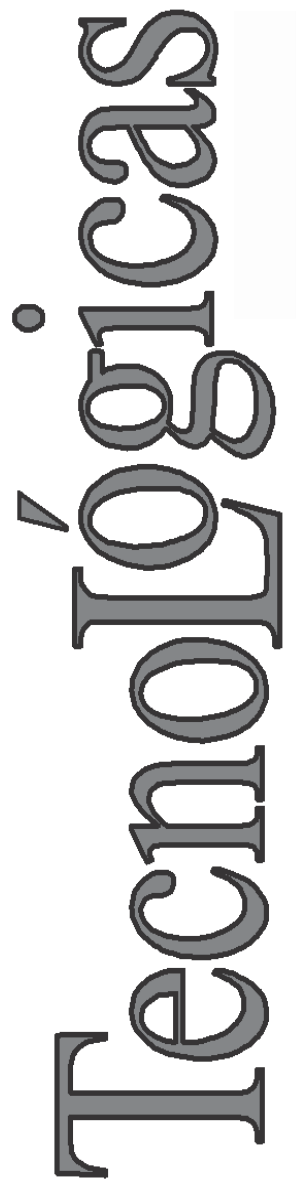

Artículo de Investigación/Research Article

\title{
Detección de Latidos Cardiacos Patológicos y Normales Utilizando Transformada por Paquetes Wavelet, Máquinas de Soporte Vectorial y Perceptrón Multicapa
}

\section{Detection of Pathological and Normal Heartbeat Using Wavelet Packet, Support Vector Machines and Multilayer Perceptron}

Alejandro J. Orozco-Naranjo ${ }^{1}$ Pablo A. Muñoz-Gutiérrez ${ }^{2}$

Recibido: 18 de abril de 2013 Aceptado: 12 de agosto de 2013

1 Ingeniero Electrónico, Grupo de Automatización y Máquinas de Aprendizaje, Facultad de Ingeniería Universidad del Quindío, Armenia-Colombia alejorozco@ingenieros.com

2 Magister en Ingeniería Eléctrica, Grupo de Automatización y Máquinas de Aprendizaje, Facultad de Ingeniería Universidad del Quindío, Armenia-Colombia pamunoz@uniquindio.edu.do 


\author{
Orozco \& Muñoz / Detección de Latidos Cardiacos Patológicos y Normales Utilizando \\ Transformada por Paquetes Wavelet, Máquinas de Soporte Vectorial y Perceptrón \\ Multicapa
}

\title{
Resumen
}

Este artículo presenta los resultados obtenidos al desarrollar una metodología para la detección de 5 tipos de latidos cardiacos (Normal (N), Bloqueo de Rama Derecha (RBBB), Bloqueo de Rama Izquierda (LBBB), Contracción Auricular Prematura (APC) y Contracción Ventricular Prematura (PVC)) utilizando la transformada por paquetes Wavelet de manera no adaptativa en la extracción de características de las señales cardiacas, empleando la función Shanon para cálculo de la entropía y adicionando una fase de identificación de nodos por cada tipo de señal cardiaca en el árbol Wavelet. La utilización de la transformada por paquetes Wavelet permite acceder a información obtenida de la descomposición tanto de baja como de alta frecuencia proporcionando un análisis más integral que el logrado con la transformada Wavelet discreta. Se evaluaron Wavelets madre de las familias Daubechies, Symlet 5 y Biortogonal inversa; que fueron resultado de una investigación previa en que se identificaron las Wavelet madre que mayor entropía presentaban con las señales cardiacas. Con la modalidad no adaptativa se reduce el costo computacional al utilizar los paquetes Wavelet, coste que representa la mayor desventaja de esta transformada, dando validez a la investigación realizada. Para la clasificación de los patrones cardiacos se emplearon las máquinas de soporte vectorial y el perceptrón multicapa. Con las máquinas de soporte vectorial empleando kernel de función de base radial, se logró un error de clasificación del 2,57 \%.

\section{Palabras clave}

Clasificación, extracción de características, latidos cardiacos, máquinas de aprendizaje supervisadas, paquetes Wavelet.

\begin{abstract}
This paper presents the results obtained by developing a methodology to detect 5 types of heartbeats (Normal (N), Right bundle branch block (RBBB), Left bundle branch block (LBBB), Premature atrial contraction (APC) and Premature ventricular contraction (PVC)), using Wavelet transform packets with non-adaptative mode applied on features extraction from heartbeats. It was used the Shannon function to calculate the entropy and It was added an identification nodes stage per every type of cardiac signal in the Wavelet tree. The using of Wavelet packets transform allows the access to information which results of decomposition of low and high frecuency, giving providing a more integral analysis than achieved by the discrete Wavelet transform. Three families of mother Wavelet were evaluated on transformation: Daubechies, Symlet and Reverse Biorthogonal, which were results from a previous research in that were identified the mother Wavelet that had higher entropy with the cardiac signals. With non-adaptive mode, the computational cost is reduced when Wavelet packets are used; this cost represents the most marked disadvantage from the transform. To classify the heartbeats were used Support Vector Machines and Multilayer Perceptron. The best classification error was achieved employing Support Vector Machine and a radial basis function; it was $2.57 \%$.
\end{abstract}

\section{Keywords}

Classification, features extraction, heartbeats, supervised learning machines, Wavelet packets. 


\section{INTRODUCCIÓN}

Con la incorporación de herramientas de procesamiento e identificación de señales al campo médico se ha facilitado el diagnóstico en un sinnúmero de especialidades, entre ellas se encuentra la cardiología, disciplina que se ocupa del comportamiento cardiaco y sus efectos en todo el organismo, por lo que es necesario el desarrollo de metodologías y técnicas que hagan menos engorroso el estudio de las señales cardiacas. Según publicación del American Cardiology College, un alto porcentaje de personas que pasan por un episodio de infarto al miocardio mueren antes de recibir atención médica especializada, casos que con una detección apropiada de los síntomas permitiría aplicar procedimientos adecuados con anticipación y así evitar desenlaces trágicos para el paciente (American Heart Association, 2001). En los últimos años se han adelantado investigaciones por parte de diversos grupos de investigación que se ocupan de la identificación de anomalías cardiacas, proponiendo la utilización de técnicas de procesamiento de señales, selección efectiva de características y clasificación de patrones (Castellanos et al., 2007; Wu et al., 2009).

En el procesamiento de bioseñales no estacionarias y en particular señales cardiacas, la transformada Wavelet Discreta (DWT) ha tenido un rol protagónico en el avance de las investigaciones, con amplia utilización en filtrado, segmentación, reducción de dimensionalidad y extracción de características (Tovar et al., 2009). A partir de la teoría de la transformada Wavelet Discreta surge la Transformada por Paquetes Wavelet (WPT), técnica que permite acceder a información omitida por la DWT y que puede contener información de interés; la obtención de esas características se hace a través de una estructura tipo árbol y emplea la entropía para conocer los nodos o ramificaciones que mejor representan la señal original (Betancourt et al., 2004). La dificultad generalizada con relación a la utilización de los paquetes Wavelet está asociada a su implementación de manera adaptativa, lo que implica un alto costo computacional si se compara con la DWT (Romo et al., 2007).

En el campo cardiaco y en especial en la clasificación de señales electrocardiográficas son pocos los trabajos que se han hecho 
empleando la transformada por paquetes Wavelet, como el documentado en Uslu et al., (2008) en que se clasificaron 6 tipos de latidos cardiacos con máquinas de soporte vectorial y redes neuronales con diferentes kernels, logrando errores de clasificación entre el $3 \%$ y 7 \%. En este caso el mejor resultado se obtuvo con el kernel función de base radial (RBF) y se contó con un total 1200 patrones para la realización de la investigación procedentes de la MIT-BIH Arrhythmya Database; en Giraldo et al., (2006) se clasifican 5 tipos de señales con una metodología por paquetes Wavelet adaptativa empleando clasificadores bayesianos y el K-ésimo vecino, los errores de clasificación estuvieron entre $5 \%$ y $10 \%$, en este se contó con un total de 250 patrones para la investigación, procedentes también de la MIT-BIH Arrhythmya Database, y los tipos de señales analizados son los mismos que se estudiaron en el presente artículo.

En la clasificación de señales electrocardiográficas suele hacerse frente a la problemática empleando clasificadores binarios o de 2 clases (Montes., et al., 2005), siendo pocos los trabajos enfocados en la multiclasificación (Giraldo et al., 2006; Uslu et al., 2008). Se desea entonces validar una metodología no adaptativa con los paquetes Wavelet para la extracción de características empleando Máquinas de Soporte Vectorial y Perceptrón Multicapa en la clasificación de los patrones cardiacos, para así aportarle al estado del arte de las alternativas disponibles en la extracción de características para la multiclasificación de patrones cardiacos y profundizar más en el análisis de las señales cardiacas considerando los antecedentes del grupo de investigación GAMA de la Universidad del Quindío, lo cuales se han enfocado en la utilización de la Transformada Wavelet Discreta (DWT).

El artículo se estructura de la siguiente manera: En la próxima sección, Métodos, se describen las principales técnicas utilizadas para la extracción de características y clasificación, en el apartado de Metodología se describe cómo se aplicaron dichas técnicas y la manera en que se hizo el tratamiento de la información; después se presentan los resultados obtenidos y finalmente las conclusiones. 


\section{MÉTODOS}

\subsection{Transformada por Paquetes Wavelet (WPT)}

Es una variación de la Transformada Wavelet Discreta (DWT). Con la DWT se descompone la señal analizada en una serie de señales restringidas a ciertas bandas de frecuencia que forman una base del espacio de funciones, cumpliendo con propiedades como ortogonalidad, tamaño, localización y suavidad. La DWT busca obtener una representación tiempo-escala de una señal discreta. La descomposición se realiza a partir de funciones más complejas, en las cuales no se varía la frecuencia sino su posición y su escala temporal, estas funciones son conocidas como Wavelet Madre, que se correlacionan con las señales originales empleando una ventana variable (Romo et al., 2007). La definición de la DWT, está dada por (1), dónde a y b son factores de escalamiento y traslación.

$C(a, b)=C_{[j, k]}=\sum_{n \in Z} f[n] \psi_{j, k}[n]$

$C_{[j, k]}$ Son coeficientes Wavelet diádicos, $f(n), \mathrm{n}=1 \ldots \mathrm{N}$ es una señal discreta y el factor diádico $j, k$ construye la Wavelet Discreta. Los coeficientes Wavelet se obtienen con algoritmos de transformación piramidal (ver Fig. 1), que corresponde a un banco de filtros pasa-bajos y filtros pasa-altas, con un sub-muestreo de factor 2 en cada nivel del banco de filtros, a estos niveles se les conoce como niveles de descomposición. Los términos producidos tras la transformación son conocidos como coeficientes de aproximación y coeficientes de detalle (Cuesta et al., 2001). Para acceder a una descomposición que represente de una manera más rica la señal analizada, la Transformada de Paquetes Wavelet descompone coeficientes normalmente no analizados, es decir los que parten de los detalles. Lo descrito se puede evidenciar claramente en la Fig. 1, donde los coeficientes de detalle de cada nivel no se descomponen, mientras los coeficientes de aproximación siguen siendo descompuestos nivel a nivel. En la Fig. 2 se evidencia la diferencia entre la DWT y WPT. Es claro que la utilización de los paquetes 
Wavelet propicia información que con la DWT no estaba disponible, información que puede incluir características discriminantes y facilite labores de clasificación. El contar con un mayor volumen de información también representa una desventaja frente a la Transformada Wavelet Discreta, porque se incrementa el costo computacional. Para disminuir los efectos de este fenómeno, se utilizan el algoritmo del Mejor Árbol (Best Tree), el cual identifica los nodos que contienen la información más relevante, valorando la entropía en cada uno de ellos con funciones o parámetros de cálculo. Los nodos se refieren a cada descomposición, en la Fig. 2, el bloque $\mathrm{AA}_{2}$ es un nodo, el bloque $\mathrm{DD}_{2}$ es otro nodo, los nodos $\mathrm{AA}_{2}$ y $\mathrm{DD}_{2}$ se encuentran en el mismo nivel de descomposición, es decir el segundo.

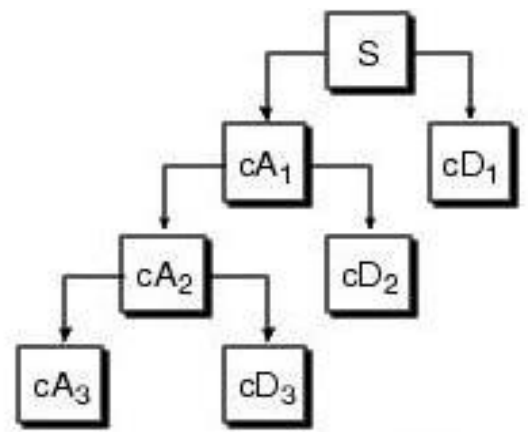

Fig. 1. Algoritmo piramidal de 3 niveles con DWT. S es la señal original. A es la salida del filtro pasa-bajos (Coeficientes de aproximación). D es la salida del filtro pasa-altas (Coeficientes de detalle). Fuente: Manual de usuario Matlab (2011)

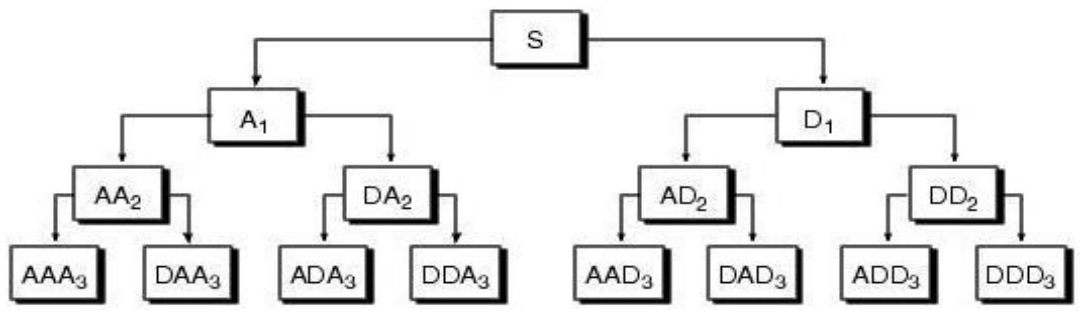

Fig. 2. Algoritmo piramidal de 3 niveles con WPT. S es la señal original. A es la salida del filtro pasa-bajos (Coeficientes de aproximación). D es la salida del filtro pasa-altas (Coeficientes de detalle). Fuente: Manual de usuario Matlab (2011) 
Generalmente los nodos son identificados a partir de algoritmos adaptativos fundamentados en la valoración hecha a partir de las funciones de entropía, por lo que aunque se disminuye el volumen de información, se produce un incremento adicional en el coste computacional adicional al ya mencionado, causado por la naturaleza adaptativa de cálculo de los mejores árboles. Uno de los parámetros más importantes al utilizar la Transformada de $\mathrm{Pa}$ quetes Wavelet es la función de cálculo de entropía para determinar el Mejor Árbol, al realizar el cálculo de entropía con las distintas funciones se busca que ésta sea lo menor posible.

\subsection{Máquinas de Soporte Vectorial}

Con las máquinas de soporte vectorial, se hace una transformación no lineal $(\Phi)$ a priori del espacio de entrada, a un espacio de características de dimensión mayor, donde posiblemente los datos pueden ser tratados de forma lineal, la Fig. 3 ilustra un poco este proceso (Muñoz et al., 2006).

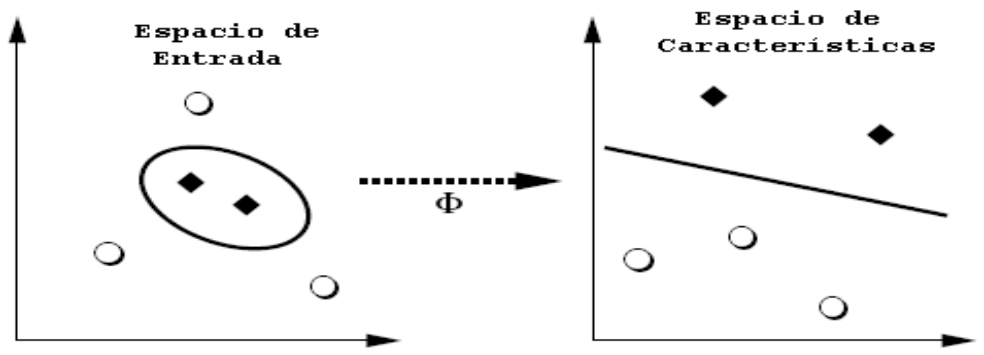

Fig. 3. Transformación no lineal a priori del espacio de entrada al espacio de características. Fuente: Autores

Las máquinas de soporte vectorial son entrenadas a través del principio de inducción de minimización del riesgo estructural, con el que se ha logrado implementar algoritmos de aprendizaje que parametricen la habilidad de generalización de las máquinas de soporte vectorial, lo que ha permitido establecer un margen máximo de separación entre las clases, además de hallar una superficie de separación entre las clases, que propicien un intervalo de confianza óptimo para el grupo de funciones clasificadoras obtenidas 
en el proceso de entrenamiento; esto a diferencia del perceptrón multicapa que es entrenado a través del principio de minimización del riesgo empírico y donde se busca la generalización a través de lograr un mínimo error de entrenamiento.

\subsection{Perceptrón Multicapa (MLP)}

El MLP es el estándar de una red neuronal artificial usada para un amplio rango de tareas, los modelos de neuronas de las MLP (Perceptrón Multicapa) son perceptrones estándares, pero su función de activación no es una función umbral o inicio lineal sino una función lineal con un gradiente. Un método muy popular de entrenamiento a través de MLP es el back-propagation o la regla de delta generalizada. El valor actual de la salida del perceptrón multicapa es calculada utilizando (2):

$$
y_{p j}=f\left(\sum_{i=0}^{n-1} w_{i} \times x_{i}\right)
$$

Los errores de clasificación del MLP son propagados desde la capa de salida a la capa de entrada, que se conoce como backpropagation. La taza de aprendizaje debe ser más grande que cero y no mayor a uno (Vapnik, 2001).

\section{METOdOLOGía}

La metodología considerada para la realización de la investigación consistió primero en disponer la información de la base de datos seleccionada, después se hizo un pre procesamiento, luego se hizo la extracción de características y finalmente se hizo la clasificación de patrones.

\subsection{Base de Datos}

La base de datos empleada, MIT-BIH Arrhythmya Database, contiene 48 horas y media de registros cardiacos, pertenecientes a 47 pacientes estudiados por el BIH Arrhythmia Laboratory, la 
duración del registro por cada paciente es de duración variable, ligeramente superior a 30 minutos. Las grabaciones fueron digitalizadas a 360 muestras por segundo por canal (siendo dos canales), con 11 bits de resolución y un rango de $10 \mathrm{mV}$. Dos cardiólogos hicieron de forma independiente las anotaciones sobre cada grabación, las anotaciones referencian el dictamen médico y la ubicación de los latidos dentro de todo el registro ECG. Las 48 grabaciones electrocardiográficas empleadas fueron tomadas en igualdad de condiciones, evitando variaciones en términos de adquisición de la señal. Se utilizó la derivación V1 por ser de alto uso en el diagnóstico médico y porque estaba disponible en la mayoría de registros. El origen de la señal tomada se encuentra en las células del músculo cardiaco, las cuales pueden ser excitadas eléctricamente (Cuesta et al., 2001). En este trabajo se trataron latidos de los tipos: Normal (N), LBBB (Bloqueo de Rama Izquierda), RBBB (Bloqueo de Rama Derecha), PVC (Contracción Prematura Ventricular), APC (Contracción Prematura Auricular).

\subsection{Pre-Procesamiento de Señales ECG}

Debido a la naturaleza de las señales electrocardiográficas, se suelen encontrar componentes ruidosos en las mismas asociados a la respiración del paciente mientras se realiza el examen, a la red eléctrica e incluso al contacto de los electrodos con la piel. Estos ruidos son conocidos como ruido de línea base, ruido de red eléctrica $(60 \mathrm{~Hz})$ y ruido térmico (Gausiano), respectivamente. Los registros electrocardiográficos deben ser filtrados para garantizar la menor presencia posible de componentes que distorsionen o afecten la señal, facilitando de esta manera el diagnóstico por parte del médico o la extracción de características. Los registros ECG de la base de datos presentan una baja presencia ruido térmico y de red eléctrica, lo que no ocurrió con el ruido de línea base.

Para el filtrado y suavizado de la señal, las técnicas basadas en la transformada Wavelet discreta resultan ser las más indicadas porque presentan una buena capacidad de adaptarse según la señal de entrada, además permiten acceder a información en tiempo y frecuencia, la metodología aplicada consiste en calcular la Transformada Wavelet Discreta de cada señal y umbralizar los 
coeficientes obtenidos, para luego con estos aplicar la Transformada Wavelet Discreta Inversa (Cuesta, 2002). En esta metodología de filtrado se deben especificar parámetros como: El tipo de umbralización, la Wavelet Madre, la estimación de ruido y el escalamiento al ruido estimado (Cuesta et al., 2001). Los parámetros empleados respectivamente fueron: Soft, Daubechies 4, Rigsure y SLN, todos determinados con asesoría médica, heurísticamente y analizando la densidad espectral de potencia del ruido filtrado. Para la eliminación de línea base se empleó una técnica se fundamentada en la utilización de un filtro de media móvil, cuya ventana depende de la cantidad total de muestras en el registro analizado (Wu et al., 2009).

Después del filtrado se deben segmentar los registros electrocardiográficos, es decir, extraer uno a uno los latidos de interés provenientes de los registros ECG; para este proceso se partió de las anotaciones de la base de datos, estas se encuentran sobre el pico representativo de cada latido cardiaco en el registro analizado, permitiendo ubicarlos en el tiempo. Gracias a la segmentación se logra contar con los latidos cardiacos, uno a uno, en archivos independientes según la anotación de interés, por ejemplo, los latidos Normales tienen la anotación 1. Antes de aplicar la segmentación a los registros ECG se determinó la cantidad de latidos de cada tipo disponibles en la base de datos, lo que llevó a la selección de 5 clases, teniendo como factor de decisión que hubiera un mínimo de 1180 ejemplos; esta cantidad limitante se definió por la cantidad disponible de latidos APC, para conocer la cantidad de latidos disponibles en los registros ECG de la base de datos, ver Tabla 1. Se tomaron 1180 ejemplos de cada tipo de latido, para un total de 5900. La metodología de segmentación consistió en buscar una anotación en particular, luego se cuenta el tiempo que hay desde el pico correspondiente a dicha anotación hasta la siguiente; de igual manera se procede para determinar el tiempo de separación entre la anotación encontrada y la anterior. Después de calcular estos tiempos, se multiplican por 0,5 y con esos límites se hace el enventanamiento del latido (Tovar et al., 2009). 
Tabla 1. Cantidad de latidos disponibles en la base de datos. Fuente: Autores

\begin{tabular}{cc}
\hline Tipo de latido & $\begin{array}{c}\text { Cantidad de latidos } \\
\text { disponibles }\end{array}$ \\
\hline Normal & 16627 \\
LBBB & 2351 \\
RBBB & 2202 \\
PVC & 2558 \\
APC & 1189 \\
\hline
\end{tabular}

\subsection{Extracción de Características}

En el proceso de extracción de características se empleó la transformada de paquetes Wavelet (WPT). Las funciones de cálculo de entropía con sus respectivos parámetros son los mostrados en la Tabla 2 por su amplio uso.

Tabla 2. Funciones y parámetros para cálculo de entropía. Fuente: Autores

\begin{tabular}{|c|c|c|}
\hline $\begin{array}{c}\text { Función de cálculo } \\
\text { de entropía }\end{array}$ & $\begin{array}{l}\text { Parámetro de la } \\
\text { función }\end{array}$ & Se define \\
\hline Shannon & - & $E(s)=-\sum s_{i}^{2} \log \left(s_{i}^{2}\right)$ \\
\hline Norm 1.5 & Potencia $(p)=1,5$ & $E(s)=\|s\|_{p}^{p}$ \\
\hline Norm 3 & Potencia $(p)=3$ & $E(s)=\|s\|_{p}^{p}$ \\
\hline Log Energy & - & $E(s)=\sum_{i} \log \left(s_{i}^{2}\right)$ \\
\hline Threshold 0,24 & $\begin{array}{l}\text { Valor de Umbral } \\
\qquad(\mathrm{p})=0,24\end{array}$ & $\begin{array}{l}E(s)=\text { La cantidad de instantes de tiempo } \\
\text { cuando la señal es mayor que el umbral p, } \\
\text { siendo } \\
\left|s_{i}\right|>p \text {; cuando }\left|s_{i}\right|<p \text { entonces } E\left(s_{i}\right)=0 .\end{array}$ \\
\hline
\end{tabular}

En funciones como Threshold y Norm, se utilizaron parámetros definidos heurísticamente. Para contar con indicadores representativos de todos los latidos cardiacos, se calculó el valor promedio de la entropía con cada función y por cada tipo de latido cardiaco. A partir de dicho valor calculado se decidió el tipo de función a utilizar. Para establecer las Wavelet Madre y cantidad de nive- 
les de descomposición, se hizo la descomposición preliminar con 7 niveles, aplicándosele a las señales de interés empleando las Wavelet Madre que mayor correlación mostraran con cada tipo de latido cardiaco, las cuales según investigación previa de los autores (Orozco et al., 2012) fueron de las familias: Daubechies, Symlet y Biortogonal Inversa. Dicha descomposición fue aplicada con cada Wavelet Madre a los 5900 latidos cardiacos filtrados y segmentados. Con los coeficientes obtenidos de la descomposición hecha en cada nodo, se hace un cálculo de entropía con la función Shannon (ver apartado de Resultados), luego se compara la diferencia entre la entropía anterior (Nodo Madre) y la sumatoria de la entropía de los nodos actuales (Nodos Hijos) que pertenecen a un mismo nivel de descomposición, por ejemplo en la Fig. 4, para los nodos hijos son Nodo 3 y Nodo 4, el Nodo Madre es el Nodo 1; cuando dicha diferencia se encuentre más cercana cero, hasta ese nivel de descomposición se aplicará la transformación.

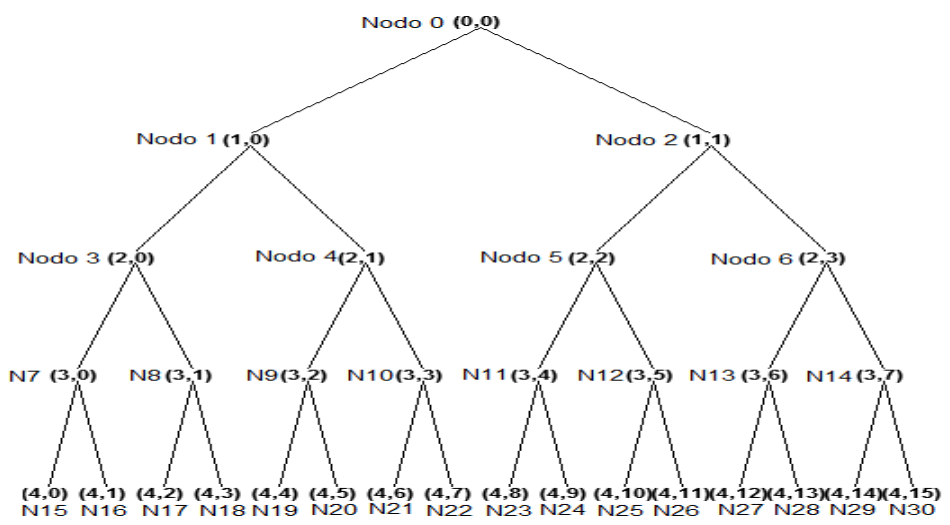

Fig. 4. Distribución y nomenclatura de los nodos obtenidos tras la descomposición de 4 niveles con la transformada por paquetes Wavelet. N: Nodo. Fuente: Autores

Después de definir la cantidad de niveles de descomposición, las Wavelet Madre y la función de entropía idóneas, se procedió a calcular el Mejor Árbol (Best Tree). Para ello se tomó una muestra de 50 latidos representativos de cada tipo de latido cardiaco, se realizó la descomposición empleando la cantidad de niveles de descomposición según la Wavelet Madre, se identificó el Mejor Árbol y registraron aquellos nodos que mejor representan a la 
señal cardiaca analizada. Es entonces cuando el modo no adaptativo aplicado a los mejores árboles de la transformada por paquetes Wavelet es considerado, obteniendo la moda de los nodos en los cálculos de los mejores árboles por cada tipo de latido cardiaco. Los nodos se encuentran distribuidos como se muestra en la Fig. 4 y se referenciarán en adelante anteponiendo la palabra nodo. Al tener identificados los nodos que resultan de interés, se procedió a hacer la extracción de características, con el fin de disponer la información que posteriormente ingresará en las máquinas de aprendizaje. Los esquemas de extracción de características consistieron en: Esquema 1: Todos los coeficientes de los nodos de interés. Esquema 2: 5 Mínimos de cada nodo de interés. Esquema 3: 5 Máximos de cada nodo de interés. Fueron evaluados otros esquemas pero los tres mencionados fueron los que mostraron mejores resultados de clasificación.

\subsection{Clasificación}

Fueron utilizadas máquinas de aprendizaje (MA) supervisadas del tipo perceptrón Multicapa (MLP) y las máquinas de soporte vectorial (SVM), las últimas se probaron con kernel Polinomial (Poly) y kernel Función de Base Radial (RBF). Para la fase de entrenamiento con cada máquina de aprendizaje se dispuso de 900 ejemplos de cada clase de latidos cardiacos, los restantes 280 se dejaron para la fase de prueba. Para la máquina de Soporte Vectorial con kernel Función de Base Radial (RBF) se utilizó Gamma=1 y $\mathrm{C}=50$. Para la Máquina de Soporte Vectorial con kernel Polinomial (Poly) se utilizó Coef=1 y Degree=3. Para el perceptrón multicapa se utilizaron 2 Capas ocultas, Peso de 50 cada capa, Función de transferencia de cada capa Tansig, 2000 Épocas y algoritmo de retro propagación de error trainscg.

\section{RESULTADOS Y DISCUSIÓN}

Como se plantea en la metodología, lo primero que se hizo fue evaluar las funciones de cálculo de entropía, esperando obtener valores idóneos cercanos a cero. En la Tabla 3, se evidencia que 
con la función de cálculo de entropía de Shannon sucede lo requerido para los 5 tipos de latidos cardiacos, por lo que fue la seleccionada. Los valores de entropía obtenidos con la función Shannon estuvieron entre 5,93 (con los latidos del tipo Normal) y 10,90 (con los latidos del tipo PVC), en general la función Shannon es la más utilizada, siendo coherente con los resultados obtenidos. Después, se estableció la cantidad de niveles adecuados de descomposición por cada Wavelet Madre a utilizar, que se muestran en la Tabla 4. Para la mayoría de las Wavelet Madre resulta adecuado emplear 4 niveles de descomposición, excepto la Daubechies 9 con 3 niveles. En trabajos de investigación similares se enfatiza en las familias Wavelet: Daubechies y Symlet; con la investigación realizada también se considera la familia Biortogonal Inversa como resultado de la validación de todas las familias Wavelet frente a los tipos de señales cardiacas estudiadas.

Tabla 3. Evaluación para cálculo de las funciones de entropía. Fuente: Autores

\begin{tabular}{ccccccc} 
& & \multicolumn{5}{c}{ Tipos de latidos cardiacos } \\
\cline { 2 - 7 } & Shannon & 5,93 & 9,83 & 5,94 & 10,90 & 7,96 \\
Funciones & Norm 1,5 & 14,58 & 23,46 & 17,59 & 27,85 & 24,63 \\
para cálculo & Threshold 0,24 & 24,71 & 40,04 & 24,66 & 56,30 & 49,01 \\
de entropía & Norm 3 & 6,72 & 12,44 & 8,96 & 13,73 & 12,74 \\
& Normal & & & & &
\end{tabular}

Posteriormente se realizó la descomposición según la cantidad de niveles de la Tabla 4 y se obtuvo el Mejor Árbol empleando la función de entropía Shannon, de donde se produjeron determinados nodos con coeficientes.

Los nodos seleccionados se muestran en la Tabla 5; la mayoría de ellos provienen de la descomposición inicial del Nodo 1, que corresponde a los coeficientes de aproximaciones del primer nivel de descomposición. El único nodo incluido en la Tabla 5 que fue obtenido a partir del Nodo 2, es decir, el de los detalles del primer nivel de descomposición, es el 11, con la Wavelet madre Daubechies 9, para mayor claridad remítase a la Fig. 4. Para evidenciar el costo computacional al emplear las metodologías adaptativa y no adaptativa con los paquetes Wavelet y la Transformada Wave- 
let Discreta, se tomó el tiempo que tarda en generarse el vector de características de un solo latido cardiaco, al ser descompuesto utilizando la Wavelet Madre daubechies 4, incluyendo todos los coeficientes de 6 nodos seleccionados para los casos adaptativo y no adaptativo, así como para la descomposición con DWT se tomaron todos los coeficientes de aproximación y detalles de los 5 primeros niveles de descomposición; con los paquetes Wavelet con metodología adaptativa el tiempo fue de $0,99 \mathrm{~s}$, con metodología no adaptativa $0,63 \mathrm{~s}$ y para la DWT $0,39 \mathrm{~s}$. La metodología no adaptativa descrita en este documento tuvo un coste intermedio, hecho importante si se compara con la metodología adaptativa, la cual es ampliamente utilizada en otros trabajos de investigación por la riqueza en información que presenta, pero supone un reto en el tratamiento del gran volumen de información generado, situación que se logra mitigar con la metodología no adaptativa.

Tabla 4. Cantidad de niveles de descomposición por cada Wavelet madre.

Fuente: Autores

\begin{tabular}{ll}
\hline \multicolumn{1}{c}{ Wavelet Madre } & Cantidad de niveles \\
\hline Daubechies 4 (db4) & 4 \\
Daubechies 9 (db9) & 3 \\
Daubechies 10 (db10) & 4 \\
Symlet 5 (sym5) & 4 \\
Biortogonal Inversa 3.1 (rbio3.1) & 4 \\
\hline
\end{tabular}

Tabla 5. Nodos a utilizar por cada Wavelet Madre. Fuente: Autores

\begin{tabular}{ll}
\hline \multicolumn{1}{c}{ Wavelet Madre } & Nodos seleccionados \\
\hline Daubechies 4 (db4) & $4 ; 7 ; 8 ; 15 ; 16 ; 17$ \\
Daubechies 9 (db9) & $3 ; 4 ; 7 ; 8 ; 9 ; 10 ; 11$ \\
Daubechies 10 (db10) & $7 ; 8 ; 9 ; 17 ; 18$ \\
Symlet 5 (sym5) & $7 ; 8 ; 15 ; 16 ; 17$ \\
Biortogonal Inversa 3,1 (rbio3,1) & $15 ; 16 ; 17 ; 18$ \\
\hline
\end{tabular}

Una vez se conocieron los nodos de interés para cada Wavelet Madre, se aplican los Esquemas 1, 2 y 3 a los coeficientes de cada nodo, para luego ingresar esta información en las máquinas de aprendizaje supervisadas para la clasificación. Los mejores resul- 
tados de clasificación con el Esquema 1, se pueden ver en la Fig. 5, donde con la Wavelet Madre Symlet 5 y la máquina de soporte vectorial con kernel RBF (función de base radial), se obtuvo un $2,57 \%$ de error porcentual, los demás resultados oscilan entre un $2,7 \%$ y $3,5 \%$. De los 5 resultados mostrados, 3 se lograron con la máquina de soporte vectorial con kernel RBF y los otros fueron con la máquina de soporte vectorial con kernel polinomial. Los mejores resultados de clasificación con el Esquema 2, se pueden ver en la Fig. 6, donde con la Wavelet Madre Daubechies 4 y la máquina de soporte vectorial con kernel $\mathrm{RBF}$, se obtuvo un 6,50\% de error porcentual, los demás resultados estuvieron entre el $6,8 \%$ y $11,7 \%$. De los 5 resultados mostrados, 3 se lograron con la máquina de soporte vectorial con kernel polinomial y 2 con la máquina de soporte vectorial con kernel RBF.

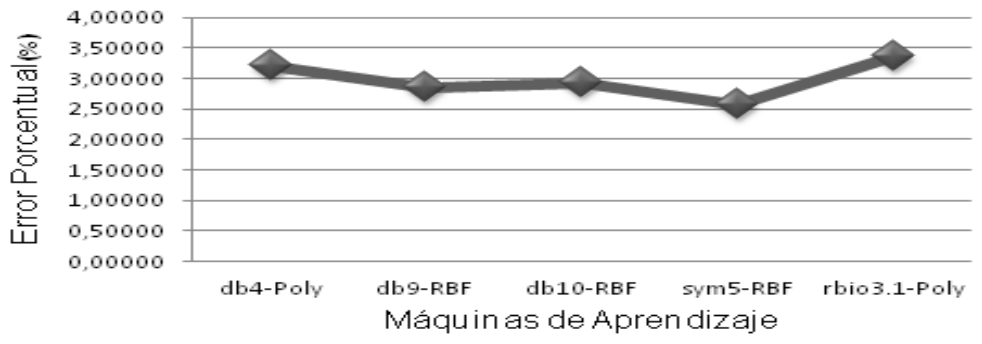

Fig. 5. Resultados de clasificación con esquema 1. Fuente: Autores

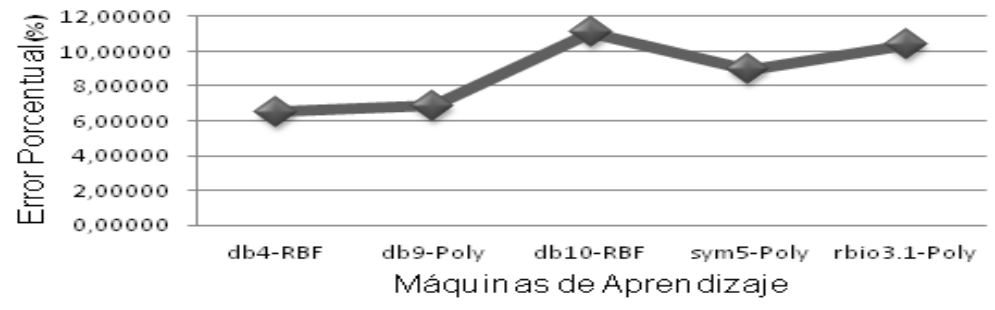

Fig. 6. Resultados de clasificación con esquema 2. Fuente: Autores

Los mejores resultados de clasificación con el Esquema 3, se pueden ver en la Fig. 7, donde con la Wavelet Madre Daubechies 4 y la máquina de soporte vectorial con kernel RBF, se obtuvo un 
5,78 \% de error porcentual. En la Fig. 8 se muestran los mejores resultados de clasificación por cada MA utilizada, para la máquina de soporte vectorial con kernel polinomial, el error porcentual fue de $3,21 \%$; con la máquina de soporte vectorial con kernel RBF, de $2,57 \%$ y con el MLP fue de 4,21\%; todos con el esquema 1 . La MSV con kernel RBF presentó mejores resultados, en particular con la Wavelet madre Symlet 5.

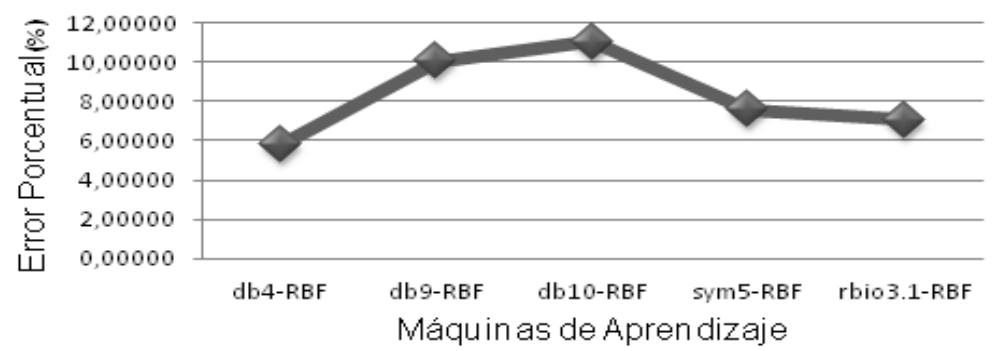

Fig. 7. Resultados de clasificación con esquema 3. Fuente: Autores

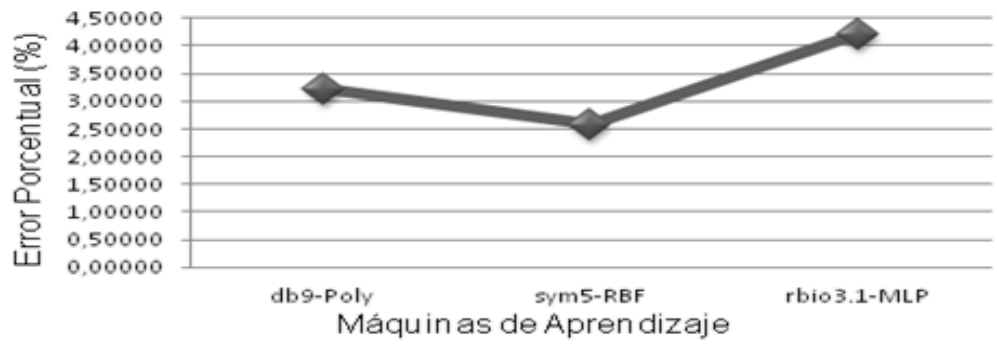

Fig. 8. Mejores resultados de clasificación por cada máquina de aprendizaje. Fuente: Autores

\section{CONCLUSIONES}

Considerando los resultados obtenidos al aplicar la Transformada por Paquetes Wavelet y seleccionando los nodos de interés a partir de las características de los tipos de las señales analizadas tanto con Máquinas de Soporte Vectorial como con el Perceptrón Multicapa, se evidencia un comportamiento satisfactorio en los resultados, estando en los mejores casos por debajo del $5 \%$ de 
error, demostrando que la Transformada por Paquetes Wavelet con la modificación hecha es una alternativa válida frente a las Transformada Wavelet Discreta (DWT), en la extracción de características de señales no estacionarias, en especial cuando las señales no analizadas por la DWT, cuentan con información de interés. En el caso cardiaco estas señales presentan un mayor representatividad en baja frecuencia pero según estudios médicos recientes, en estudios médicos recientes se han identificado comportamientos de alta frecuencia que son de interés para el personal médico. Con las máquinas de soporte vectorial se obtuvieron los resultados de clasificación más satisfactorios, con errores porcentuales que estuvieron entre $2,5 \%$ y $3,2 \%$; este mismo comportamiento se presentó con la Transformada Wavelet Discreta en investigaciones anteriores al analizar las mismas señales cardiacas. Las máquinas de soporte vectorial con kernel RBF presentan una alta compatibilidad con paquetes Wavelet, puesto que suponen distribuciones de la información concentradas en determinadas áreas de la transformación en espacios, lograda con las máquinas de aprendizaje. Es de interés realizar otros estudios en que se valide la Transformada con Paquetes Wavelet de manera no adaptativa con otro tipo de señales en la extracción de características, se apliquen algoritmos de aprendizaje para identificación de mejores nodos en los árboles Wavelet y se haga una validación con máquinas de aprendizaje no supervisadas.

\section{REFERENCIAS}

American Heart Association (2001). Handbook of emergency cardiovascular care for health team, 20-24. Dallas, USA.

Betancourt, G., Giraldo, E., Franco, J. (2004). Reconocimiento de patrones de movimiento a partir de señales electromiográficas. Scientia et Técnica, 26, 53-58.

Castellanos, C. G., Godino, J. I., Delgado, E. (2007). Análisis acústico sobre señales de auscultación digital para la detección de soplos cardíacos. Avances en sistemas e informática, 4(3), 171-182.

Cuesta, F., Novak, D., ECK, D., Pérez, V., Andreu, J. (2001). Electrocardiogram baseline removal using Wavelet approximations. Libro de Actas XVIII Congreso Anual de la Sociedad de Ingeniería Biomédica, CASEIB, 103-106. Cartagena, Colombia. 
Cuesta, F. (2002). Estudio de métodos para procesamiento y agrupación de señales electrocardiográficas. Tesis doctoral, Universidad Politécnica de Valencia, Departamento de Informática de Sistemas y Computadoras (DISCA), 69-114, España.

Kharate, G. (2010). Color image compression based on Wavelet packet Best Tree. International Journal of Computer Science Issues, IJCSI, 7(2), 31-35.

Manual de Usuario Matlab (R). "Wavelet Packets" Mathworks, Cambridge MA. 2011.

Montes, V., Guarín, C., Castellanos, G. (2005). Extracción de características de ECG basadas en transformaciones no lineales y Wavelets. Ingeniería e investigación, 25 (3), 39-48.

Muñoz, P.A., Cardona, J., Ibarguen F. (2006). Máquinas de vectores de soporte: un enfoque teórico. Revista de Investigaciones Universidad del Quindio, 16, 107-120.

Orozco, A. J., Muñoz P.A. (2012). Selección de Wavelets Madre para la caracterización de 5 tipos de latidos cardiacos empleando la Transformada Wavelet Discreta. Revista de Investigaciones de la Universidad del Quindio, 23, 71-80.

Romo, H., Realpe, J., Jojoa, P. (2007). Análisis de señales EMG superficiales y su aplicación en control de prótesis de mano. Avances en Sistemas e Informática, 4(1), 127-136.

Tovar, D.A., Orozco, A.J., Munoz, P.A. (2009). Transformada Wavelet y máquinas de soporte vectorial para la identificación de arritmias. Revista de Investigaciones Universidad del Quindio, 19, 104-114.

Uslu, E., Bilgin, G. (2008). Classification of heart arrthymias by using Wavelet and merged Wavelet Packet Transforms, signal processing. Communication and Applications Conference 2008. SIU. IEEE, 4, 2022.

Vapnik, V. (2001). The nature of statistical learning theory. $2^{\text {nd }}$ Edition, New York, USA, Springer Verlag Inc., 254-313,

Wu, Y.F., Rangayyan, R.M., Zhou, Y.C., Ng, S.C. (2009). Filtering electrocardiographic signals using an unbiased and normalized adaptive noise reduction system. Med Eng Phys, 31(1), 17-26. 